\title{
Eine Modellerkrankung mit geschlechtsspezifischer Relevanz
}

\author{
Katrin Rauen a,b,c, Susanne Walitza ${ }^{\mathrm{d}, e}$, Egemen Savaskan ${ }^{\mathrm{f}, \mathrm{b}}$, Erich Seifritz $^{\mathrm{f}, \mathrm{g}}$ \\ ${ }^{a}$ Dr. med., FEBN, Fachärztin für Neurologie; ${ }^{b}$ Klinik für Alterspsychiatrie, Psychiatrische Universitätsklinik Zürich; ${ }^{c}$ Institut für Schlaganfall und

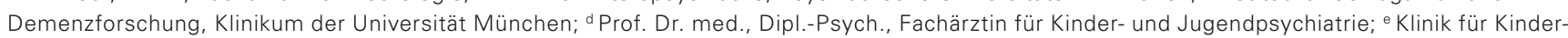 \\ und Jugendpsychiatrie und Psychotherapie, Psychiatrische Universitätsklinik Zürich; ${ }^{\dagger}$ Prof. Dr. med., Facharzt für Psychiatrie und Psychotherapie; \\ ${ }^{9}$ Klinik für Psychiatrie, Psychotherapie und Psychosomatik, Psychiatrische Universitätsklinik Zürich
}

Frauen leiden nach der Pubertät zweimal häufiger unter Depressionen als Männer. Ob dieses Ungleichgewicht real ist oder das «männliche» Depressionssyndrom bisher unzureichend erkannt wird, ist unklar. Biomarker könnten helfen, diese diagnostische Lücke bereits hausärztlich zu schliessen und dadurch auch das depressionsassoziierte Demenzrisiko zu senken.

Die unipolare Depression wird von der WHO weltweit als dritthäufigste Ursache für krankheitsbedingte Belastung identifiziert und den «Global Burden of Disease»-Report bis 2030 vor Herzerkrankungen und Demenzen anführen [1, 2]. In der Schweiz leiden 7\% der Bevölkerung an einer behandlungsbedürftigen unipolaren Depression [3], und bereits während der Jugend sind Depressionen für die meisten verlorenen Lebensjahre (DALYs) verantwortlich. Die Relevanz der Erkrankung wird durch die Eingruppierung der WHO in die höchste Behinderungskategorie - zusammen mit Psychose, schwerer Migräne, Tetraplegie und Krebs im Endstadium - deutlich [1]. Während Depressionen im Kindesalter zwischen beiden Geschlechtern noch weitestgehend gleichmässig verteilt sind und im Vergleich zum Jugend- und Erwachsenenalter eher unspezifische, somatische Beschwerden überwiegen (s.u.), leiden im Alter von zwölf Jahren weibliche Jugendliche 2,4-mal häufiger an einer schweren Depression als gleichaltrige männliche Jugendliche. Im Alter von 13 bis 16 Jahren wird das Maximum mit einem dreifach

Im Alter von 13 bis 16 Jahren wird das Maximum mit einem dreifach erhöhten Risiko bei jungen Frauen erreicht.

erhöhten Risiko bei jungen Frauen im Vergleich zu gleichaltrigen Männern erreicht [4]. Über die weitere Lebensspanne leiden Frauen konstant zweimal häufiger an Depressionen als Männer [5]. Ob diese geschlechtsspezifische Prävalenz über die Lebensspanne real ist oder auf einem kriteriumsbezogenen Ge- schlechterbias beruht, ist Gegenstand der aktuellen Diskussion [5]. Argumente für einen Geschlechterbias stützen sich auf die Annahme, dass die Validität der Klassifizierungssysteme (DSM-5/ICD-10) zur Detektion der geschlechtsspezifischen Unterschiede infrage gestellt wird, da die "weiblichen» Depressionssymptome überrepräsentiert sind, die depressive Symptomatik bei Männern leichter übersehen wird und die Syndromdiagnose auf der individuell erhobenen Psychopathologie basiert [5]. Um diese Frage objektiv beantworten zu können, ist es notwendig, die Psychopathologie geschlechtsspezifisch besser zu verstehen und geschlechtsunabhängige Biomarker in der Diagnostik zu implementieren.

\section{Geschlechtsspezifische Psychopathologie}

Die sogenannte «weibliche» Depression umfasst, dass Frauen ihre Gefühle häufiger internalisieren, mehr grübeln, unter Schuld- sowie Insuffizienzgefühlen leiden, ihre Symptome eher wahrnehmen und professionelle Hilfe suchen. Gewichtszunahme, bulimisches Verhalten, Angst, Hypersomnie und somatoforme Störungen zählen zu den typischen weiblichen Zeichen einer atypischen Depression [4-10]. Dagegen fallen Männer durch Reizbarkeit, Agitation, Aggressivität mit Impulskontrollstörung und Substanzkonsum auf [11]. Allerdings gibt es auch Evidenz für erhöhte Reizbarkeit als Zeichen der Chronifizierung und Depressionsschwere bei depressiven Frauen [5, 12,13]. Bereits in der kindlichen Entwicklung spielt «Reizbarkeit» eine zentrale Rolle, denn bis zu 51\% der irritablen Kinder lei- 
den als Erwachsene an einer schweren Depression [14]. Die frühe Manifestation der Irritabilität und Reizbarkeit in Kombination mit einer depressiven Stimmung erhöht das Suizidrisiko in der Adoleszenz bei beiden Geschlechtern [15], weshalb diesem Symptom über die gesamte Lebensspanne mehr Beachtung gebührt. «Reizbarkeit» kann auch ein Kriterium der bipolaren Störung sein, so dass neben der geschlechtsspezifischen auch die differentialdiagnostische Abgrenzung relevant ist und Biomarker für die frühe hausärztliche und objektive Diagnostik unabdingbar sind.

\section{Soziale Signal-Transduktions-Theorie}

Diese Theorie geht von multifaktoriellen Mechanismen aus, die durch ungünstige soziale Umwelterfahrungen in der frühen Kindheit/Jugend für die Entstehung der Inflammation als Risikofaktor der Depression relevant sind [16, 17]. Progesteron, Testosteron und hohe Östrogenspiegel gelten hinsichtlich einer depressiven Erkrankung als anti-inflammatorisch und protektiv [18]. Dagegen sind niedrige Östrogenspiegel, wie sie in hormonellen Übergangsperioden - post-partum und peri-menopausal - vorkommen, pro-inflammatorisch und schädlich $[17,19,20]$. Sie erklären zumindest teilweise das erhöhte Risiko für Frauen nach der Geburt und während der Menopause, an einer Depression zu leiden. Die beeinträchtigte psychische Gesundheit post-partum betrifft nicht nur die Mütter selbst, sondern führt zu einem um $20 \%$ höheren Risiko für ein unsicheres Bindungsverhalten ihrer Kinder [21], welches per se mit einem erhöhten Risiko für Angst und Depression einhergeht. Die frühe sozial-emotionale Entwicklung des Säuglings ist von Umgebungsfaktoren und der Bindung zur Primärperson abhängig, weshalb der Diagnostik von Post-partum-Depressionen eine entscheidende und generationenübergreifende Rolle zukommt [22]. Väter scheinen durch gute Beziehungsgestaltung das Bindungsdefizit ihrer Kinder zur depressiven Mutter ausgleichen zu können [23]. Ob mütterliche Depressionen Mädchen und Jungen in ihrer Entwicklung unterschiedlich betreffen, ist unklar und könnte ein wegweisender Faktor für die erhöhte Vulnerabilität von Mädchen/jungen Frauen in der (Vor-)Pubertät sein.

\section{Biomarker}

Biomarker spielen in der klinischen Versorgung bisher keine Rolle und sind in der aktuellen S3-Leitlinie «Unipolare Depression» der Deutschen Gesellschaft für Psychiatrie und Psychotherapie, Psychosomatik und Nervenheilkunde (DGPPN) nicht erwähnt [5]. Bisherige Forschungsergebnisse deuten auf einen altersunab- hängigen und kausalen Zusammenhang zwischen Inflammation und Depression [24] sowie auf teilweise geschlechtsspezifische Biomarker-Profile hin [25]. Vielversprechende Blut-Biomarker sind erhöhte CRP- und IL-6-Spiegel [24-26], Carbonylproteine (oxidativer Stress), erniedrigter BDNF-Wachstumsfaktor sowie IL10, IL-17A, IL-4 und TNF-a [27, 28]. Neben der frühen hausärztlichen Diagnostik wären Blut-Biomarker wertvoll, um die Ansprechrate einer antidepressiven Therapie zu objektivieren oder vorauszusagen. Diese Forschung ist bereits gut etabliert, die Umsetzung steckt aber noch in den Kinderschuhen, so dass bisher keine Empfehlungen für die klinische Routine existieren.

\section{Demenzrisiko}

Die Frage, ob schwere Depressionen das Demenzrisiko erhöhen, kann aufgrund der aktuellen Studienlage bejaht werden [29]. Dabei ist das Erkrankungsalter bei Erstmanifestation der affektiven Störung relevant: Depressive Episoden im jungen Erwachsenenalter verdoppeln und Episoden im mittleren bis höheren Lebensalter verfünffachen das Demenzrisiko [30]. Aus der Framingham-Studie wissen wir, dass höheres Alter, weibliches Geschlecht, geringere Schulbildung und Depression das Risiko für eine Alzheimer-Demenz erhöhen [31]. Depressionen im mittleren bis hohen Alter sind mit einem fast vierfach erhöhten Risiko für eine vaskuläre Demenz und einem zweifach erhöhten Risiko für eine Alzheimer-Demenz assoziiert, wobei rezidivierende depressive Episoden eine vaskuläre Demenz begünstigen [32]. Da Frauen doppelt so häufig wie Männer von schwerer Depression und Alzheimer-Demenz betroffen sind, müssen geschlechtsspezifische Faktoren in Forschung und Klinik fokussiert werden, um den mutmasslichen Zusammenhang zwischen Depression und Demenz in Längsschnittstudien nachzuweisen.

Insgesamt stehen wir am Anfang der geschlechtsspezifischen Biomarkerforschung, die durch zahlreiche Faktoren (Hormonstatus, Alter, Body Mass Index) und Stressoren mit resultierender Variabilität auf Genom-, Epigenom-, Transkriptom- oder Proteom-Ebene [33-35] multizentrische Forschung mit Big-Data-Analysen erfordert, um das Leiden unserer Patienten und Patientinnen best- und frühestmöglich erkennen und behandeln zu können.

\footnotetext{
Literatur

1 WHO. The global burden of disease: 2004 update 2008

(Available from: https://www.who.int/healthinfo/global_burden disease/2004_report_update/en/).

2 Malhi GS, Mann JJ. Depression. Lancet. 2018;392:2299-312.

3 Baer N, Schuler D, Füglister-Dousse S, Moreau-Gruet F. Depressionen in der Schweiz. Daten zur Epidemiologie, Behandlung und
} 
sozial-beruflichen Integration. Neuenburg: Schweizerisches Gesundheitsobservatorium, Obsan Bericht 56; 2013.

4 Salk RH, Hyde JS, Abramson LY. Gender differences in depression in representative national samples: Meta-analyses of diagnoses and symptoms. Psychol Bull. 2017;143:783-822.

5 DGPPN B, KBV, AWMF (Hrsg.) für die Leitliniengruppe Unipolare Depression. S3-Leitlinie/Nationale VersorgungsLeitlinie Unipolare Depression - Langfassung. 2015

6 Vetter JS, Spiller TR, Cathomas F, Robinaugh D, Brühl A, Boeker H, in a treatment-seeking population - a cross-sectional study. J Affect Disord. 2020;278:357-64.

7 Seeman MV. Psychopathology in women and men: focus on female hormones. Am J Psychiatry. 1997;154:1641-7.

8 Marcus SM, Kerber KB, Rush AJ, Wisniewski SR, Nierenberg A, Balasubramani GK, et al. Sex differences in depression symptoms in treatment-seeking adults: confirmatory analyses from the Sequenced Treatment Alternatives to Relieve Depression study. Compr Psychiatry. 2008;49:238-46.

9 Blanco C, Vesga-López O, Stewart JW, Liu SM, Grant BF, Hasin DS. Epidemiology of major depression with atypical features: results from the National Epidemiologic Survey on Alcohol and Related Conditions (NESARC). J Clin Psychiatry. 2012;73:224-32.

10 Dekker J, Koelen JA, Peen J, Schoevers RA, Gijsbers-van Wijk C. Gender differences in clinical features of depressed outpatients: preliminary evidence for subtyping of depression? Women Health. 2007;46:19-38.

11 Winkler D, Pirek E, Kasper S. Anger attacks in depression - evidence for a male depressive syndrome. Psychother Psychosom. 2005;74:303-7.

12 Judd LL, Schettler PJ, Coryell W, Akiskal HS, Fiedorowicz JG. Overt irritability/anger in unipolar major depressive episodes: past and current characteristics and implications for long-term course. JAMA Psychiatry. 2013;70:1171-80.

13 Perlis RH, Fraguas R, Fava M, Trivedi MH, Luther JF, Wisniewski SR, et al. Prevalence and clinical correlates of irritability in major depressive disorder: a preliminary report from the Sequenced Treatment Alternatives to Relieve Depression study. J Clin Psychiatry. 2005;66:159-66; quiz 47:273-4.

14 Eyre O, Hughes RA, Thapar AK, Leibenluft E, Stringaris A, Davey Smith G, et al. Childhood neurodevelopmental difficulties and risk of adolescent depression: the role of irritability. J Child Psychol Psychiatry. 2019;60:866-74. Association of Childhood Irritability and Depressive/Anxious Mood Profiles With Adolescent Suicidal Ideation and Attempts. JAMA Psychiatry. 2018;75:465-73.

16 Slavich GM, Irwin MR. From stress to inflammation and major depressive disorder: a social signal transduction theory of depression. Psychol Bull. 2014;140:774-815.

17 Slavich GM, Sacher J. Stress, sex hormones, inflammation, and major depressive disorder: Extending Social Signal Transduction Theory of Depression to account for sex differences in mood disorders. Psychopharmacology (Berl). 2019;236:3063-79.

18 Klein SL. The effects of hormones on sex differences in infection: from genes to behavior. Neurosci Biobehav Rev. 2000;24:627-38.

19 Freeman EW, Sammel MD, Lin H, Nelson DB. Associations of hormones and menopausal status with depressed mood in women with no history of depression. Arch Gen Psychiatry. 2006;63:375-82.

Dr. med. Katrin Rauen, FEBN Klinik für Alterspsychiatrie Psychiatrische Universitätsklinik Zürich Minervastrasse 145 CH-8032 Zürich katrin.rauen[at]uzh.ch dritthäufigste Ursache für krankheitsbedingte Belastung identifiziert.

- Frauen leiden nach der Pubertät zweimal häufiger unter Depressionen als Männer.

- Ob diese geschlechtsspezifische Prävalenz über die Lebensspanne real ist oder auf einem kriteriumsbezogenen Geschlechterbias beruht, ist Gegenstand der aktuellen Diskussion.

- Die Frage, ob schwere Depressionen das Demenzrisiko erhöhen, kann bejaht werden.

- Insgesamt stehen wir am Anfang der geschlechtsspezifischen Biomarkerforschung. et al. Sex differences in depressive symptoms and their networks

15 Orri M, Galera C, Turecki G, Forte A, Renaud J, Boivin M, et al.

20 Frokjaer VG, Pinborg A, Holst KK, Overgaard A, Henningsson S, Heede M, et al. Role of Serotonin Transporter Changes in Depressive Responses to Sex-Steroid Hormone Manipulation: A Positron Emission Tomography Study. Biol Psychiatry. 2015;78:534-43.

21 Barnes J, Theule J. Maternal depression and infant attachment security: A meta-analysis. Infant Ment Health J. 2019;40:817-34.

22 Behrendt HF, Scharke W, Herpertz-Dahlmann B, Konrad K, Firk C. Like mother, like child? Maternal determinants of children's early social-emotional development. Infant Ment Health J. 2019;40: 234-47.

23 Malmberg LE, Lewis S, West A, Murray E, Sylva K, Stein A. The influence of mothers' and fathers' sensitivity in the first year of life on children's cognitive outcomes at 18 and 36 months. Child Care Health Dev. 2016;42:1-7.

24 Valkanova V, Ebmeier KP, Allan CL. CRP, IL-6 and depression: a systematic review and meta-analysis of longitudinal studies. J Affect Disord. 2013;150:736-44.

25 Strawbridge R, Arnone D, Danese A, Papadopoulos A, Herane Vives A, Cleare AJ. Inflammation and clinical response to treatment in depression: A meta-analysis. Eur Neuropsychopharmacol. 2015;25:1532-43.

26 Smith KJ, Au B, Ollis L, Schmitz N. The association between C-reactive protein, Interleukin- 6 and depression among older adults in the community: A systematic review and meta-analysis. Exp Gerontol. 2018;102:109-32.

27 Bocchio-Chiavetto L, Bagnardi V, Zanardini R, Molteni R, Nielsen MG, Placentino A, et al. Serum and plasma BDNF levels in major depression: a replication study and meta-analyses. World J Biol Psychiatry. 2010;11:763-73.

28 Wollenhaupt-Aguiar B, Librenza-Garcia D, Bristot G, Przybylski L, Stertz L, Kubiachi Burque R, et al. Differential biomarker signatures in unipolar and bipolar depression: A machine learning approach. Aust N Z J Psychiatry. 2020;54:393-401.

29 Livingston G, Huntley J, Sommerlad A, Ames D, Ballard C, Banerjee S, et al. Dementia prevention, intervention, and care: 2020 report of the Lancet Commission. Lancet. 2020;396:413-46.

30 Byers AL, Yaffe K. Depression and risk of developing dementia. Nat Rev Neurol. 2011;7:323-31.

31 Saczynski JS, Beiser A, Seshadri S, Auerbach S, Wolf PA, Au R. Depressive symptoms and risk of dementia: the Framingham Heart Study. Neurology. 2010;75:35-41.

32 Barnes DE, Yaffe K, Byers AL, McCormick M, Schaefer C, Whitmer RA. Midlife vs late-life depressive symptoms and risk of dementia: differential effects for Alzheimer disease and vascular dementia. Arch Gen Psychiatry. 2012;69:493-8.

33 Strawbridge R, Young AH, Cleare AJ. Biomarkers for depression: recent insights, current challenges and future prospects. Neuropsychiatr Dis Treat. 2017;13:1245-62.

34 Begliuomini S, Casarosa E, Pluchino N, Lenzi E, Centofanti M, Freschi L, et al. Influence of endogenous and exogenous sex hormones on plasma brain-derived neurotrophic factor. Hum Reprod. 2007;22:995-1002.

35 Soares CN. Depression and Menopause: Current Knowledge and Clinical Recommendations for a Critical Window. Psychiatr Clin North Am. 2017;40:239-54.

\section{L'essentiel en bref}

- Selon I'OMS, la dépression unipolaire est la troisième cause la plus fréquente de détresse liée à la maladie dans le monde.
Les femmes sont deux fois plus susceptibles de souffrir de dépression après la puberté que les hommes.

- La question de savoir si cette prévalence spécifique au sexe sur toute la durée de vie est réelle ou si elle est fondée sur un biais sexiste fait actuellement l'objet de débats.

- Une dépression majeure peut augmenter le risque de démence.

- Actuellement, la recherche sur les biomarqueurs de genre en est à ses prémices. 